Published in final edited form as:

Trends Biotechnol. 2015 September ; 33(9): 525-533. doi:10.1016/j.tibtech.2015.06.009.

\title{
piggyBac-ing models and new therapeutic strategies
}

\author{
Lauren E. Woodard ${ }^{1,2}$ and Matthew H. Wilson ${ }^{1,2, *}$ \\ ${ }^{1}$ Department of Veterans Affairs, Tennessee Valley Health System, Nashville, TN, USA \\ ${ }^{2}$ Department of Medicine, Division of Nephrology and Hypertension, Vanderbilt University Medical \\ Center, Nashville, TN, USA
}

\begin{abstract}
DNA transposons offer an efficient non-viral method of permanently modifying the genomes of mammalian cells. The piggyBac transposon system has proven effective in genomic engineering of mammalian cells for pre-clinical applications including gene discovery, simultaneous multiplexed genome modification, animal transgenesis, gene transfer in vivo achieving long-term gene expression in animals, and for genetic modification of clinically relevant cell types including induced pluripotent stem cells and human T lymphocytes. piggyBac has many desirable features including seamless excision of transposons from the genomic DNA and the potential to target integration events to desired DNA sequences. This review explores these recent applications and also highlights the unique advantages of using piggyBac for developing new molecular therapeutic strategies.
\end{abstract}

\section{Keywords}

piggyBac; transposon; iPSC; transgenesis; cell therapy; immunotherapy

\section{piggyBac: a versatile tool for biotechnology}

DNA-based transposon systems first emerged as efficient tools for genome engineering of mammalian cells after the Sleeping Beauty transposon system was resurrected from the genome of the medaka fish [1]. Transposon DNA vectors can be engineered for a variety of purposes including transgenesis, gene therapy, gene trapping, or insertion of other DNA elements into the genomes of cells (see Glossary). The piggyBac transposon system is naturally active and was first discovered in insect cells while propagating Baculovirus in the TN-386 cell line, from the cabbage looper moth Trichoplusia $n i$ of the order Lipodoptera [2, 3]. The cause of unexpected viral plaques was the insertion of novel Class II Mobile DNA elements in the Baculovirus. The inserted mobile DNA was being carried "piggyback" by the virus, so it was named piggyBac; the capitalized and shortened "Bac" part of the name signifies its Baculovirus-related discovery. Since its discovery in 1983, piggyBac (formerly

\footnotetext{
*Corresponding author: Matthew H. Wilson matthew.wilson@ vanderbilt.edu.

Publisher's Disclaimer: This is a PDF file of an unedited manuscript that has been accepted for publication. As a service to our customers we are providing this early version of the manuscript. The manuscript will undergo copyediting, typesetting, and review of the resulting proof before it is published in its final citable form. Please note that during the production process errors may be discovered which could affect the content, and all legal disclaimers that apply to the journal pertain.
} 
named IFP-2) was studied mostly in insects. piggyBac was first shown to be efficient in gene transfer in mice in 2005 [4]. Since that time, it has been used for genomic modification of human cells [5] and for a variety of applications. These include mammalian transgenesis, mutagenesis, ex vivo modification of clinically relevant cell types, and gene transfer in mammals in vivo. Such applications have opened new areas of research that will hopefully lead to new therapeutic strategies for human disease.

piggyBac-based gene transfer or mobilization is carried out through a "cut and paste" mechanism (Box 1). For most applications, the piggyBac transposase and piggyBac transposon are carried on two separate plasmids (trans). It is also possible to deliver the transposase and transposon on the same plasmid ( $\mathrm{cis}$ ) with the transposase gene encoded outside of the transposon inverted terminal repeat elements (IRs). When the piggyBac transposase protein is expressed in mammalian cells, it binds to the inverted repeats of the transposon, nicking the DNA and freeing a 3' hydroxyl group at both ends of the transposon. This results in hydrophilic attack of the flanking TTAA sequence and hairpin formation (Box 1), freeing the transposon from its plasmid backbone [6]. The plasmid backbone is then repaired by host cell factors by ligation of the complementary TTAA overhangs. piggyBac transposase locates TTAA sequences in the genomic DNA of the mammalian cells. Through hairpin resolution of the transposon and hydrophilic attack of the genomic DNA by 3' hydroxyl groups on the transposon, a staggered 4-bp cut in the genomic DNA is produced, creating a transient double-strand break with TTAA overhangs on both sides of the break. The transposon is then inserted into the genomic DNA at the TTAA site, resulting in a duplication of this TTAA such that a TTAA is found on both sides of the transposon. Upon excision of the transposon by piggyBac transposase, which can be induced and selected for to rid the cells of the transgene, the single stranded TTAAs are religated to reform a single TTAA. Thus, the unique mechanism of piggyBac transposition results in a unique advantage: seamless excision of the transposon sequence (Box 2). This phenomenon has been put to great advantage already to generate transgene-free induced pluripotent stem (iPS) cells [7, 8]. The piggyBac transposon system has proven important for a number of versatile biotechnology applications (Figure 1). piggyBac enables greater cargo capacity compared to commonly used viral vectors for gene transfer and it has a different genomic target sequence, a higher likelihood of hitting genes, and does not suffer from overproduction inhibition as compared to Sleeping Beauty (Table 1).

\section{Gene discovery via insertional mutagenesis}

piggyBac has been used for insertional mutagenesis to evaluate various pathways both in tissue culture ex vivo and in live animals in vivo. Transposon integrations can be recovered and mapped to identify genes that have been misregulated (either activated or inactivated), resulting in the phenotype of the mutant cell or organism [9]. As piggyBac is known to have different genomic target site preferences than viruses or other transposon systems, it has proven to be a new valuable tool for gene discovery [9]. The ability to turn on mutagenesis in transgenic animals with exquisite control over the timing and cell type of interest has led to the discovery of many new oncogenes and tumor suppressors [9]. 
Insertional mutagenesis is a powerful way to elucidate genes involved in a variety of pathways in cultured cells. In vitro, piggyBac has been used for gene discovery in both mouse neural stem cells [10] and human pancreatic cells [11]. Studies in mouse embryonic stem (mES) cells have included genetic manipulation of Blm-deficient mES cells [12] and inducible genomic transposition in $\mathrm{mES}$ cells for gene trapping [13].

piggyBac transposons were used to insert loxP sites into the mouse genome whereby subsequent expression of the Cre recombinase would result in large chromosomal deletions in intact animals [14]. Reporter-gene trapping mouse lines [15] and cancer gene discovery lines [9] have been generated to aid in gene discovery, identifying genes that were not previously identified by retroviral or Sleeping Beauty transposon screens. Additionally, piggyBac and Sleeping Beauty may be used in a combinatorial manner if desired as transposons contain both sets of inverted repeats [9]. Recently, a conditional piggyBac system was employed to identify novel genes in pancreatic cancer, revealing the power of the versatile transposon system in identifying new cancer genes that could be applied to a variety of tissues and cancer types [16].

\section{Generation of transgenic animals}

Animal transgenesis represents an important methodology for discovering and evaluating disease processes and establishing pre-clinical models of human diseases. One of the limiting factors for generation of traditional transgenic animals is the low efficiency of generating them. The increased efficiency of transgenesis after employing piggyBac can make such manipulations more efficient and affordable. piggyBac was used to make transgenic mice that expressed reporter genes [4]. Subsequent studies have also used piggyBac to genetically modify mouse embryonic (ES) cells $[12,17,18]$. Rat induced pluripotent stem cells [19] have also been modified by piggyBac to make transgenic animals [20]. Pig cells have also been gene-modified using piggyBac to make transgenic animals $[21,22]$. Animal transgenesis remains an important research endeavor for modelling human disease.

\section{Engineering stable cell lines}

piggyBac offers a versatile plasmid based system for stable cell line generation, especially for generation of cultured cells stably expressing one or more recombinant gene sequences for biomedical research, biotechnology, or drug development. More traditional methods of transfecting plasmids and using antibiotic selection are not efficient (Figure 2) and have severe limitations especially when engineering cells to co-express multiple transgenes or multi-protein complexes. Multiplexed piggyBac transposon delivery was by co-expressing large transgenes encoding a multi-subunit neuronal voltage-gated sodium channel (SCN1A) containing a pore-forming subunit and two accessory subunits, plus two additional genes for selection and obtained robust sodium current through 38 passages suitable for use on an automated high-throughput electrophysiology platform [23]. Three large (up to $10.8 \mathrm{~kb}$ ) piggyBac transposons were co-delivered, thereby generating a heterozygous SCN1A stable cell line with robust functional expression. Others have also generated vectors capable of 
modular assembly such that multiple transgenes can be co-integrated into cells using a single piggyBac vector [24].

Investigators have demonstrated the ability of piggyBac to generate stable cell lines for large-scale protein production [25, 26]. Engineered doxycycline inducible stable cells lines have proven capable of reproducible protein production in the absence of selection, even the ability to produce $750 \mathrm{mg}$ of an endoplasmic-reticulum-resident fucosyultransferase.

Investigators have also used piggyBac transposons with inducible expression for myogenic differentiation of human iPSCs including human a human myopathy [27] and to reliably incorporate BAC transgenesis in human embryonic stem cells [28]. Therefore, the plasmid based piggyBac system offers a capable transgene delivery system for single or simultaneous multi-gene modification of cultured cells for a variety of research applications, including potential new therapies (Box 3).

\section{Modification of clinically relevant cells}

Cell therapy strategies are emerging for a variety of human diseases. piggyBac has been used to generate mouse and human induced pluripotent stem cells (iPSC) and to modify human iPSCs, human embryonic stem (hES) cells, human hematopoietic stem cells (HSCs), and human T lymphocytes.

\section{Cellular reprogramming of iPS cells}

iPSCs were first generated by using a combination of transcription factors to reprogram mammalian cells to a stem cell-like phenotype $[29,30]$. The factors were discovered by narrowing a pool of 15 retroviral vectors, each carrying a different transcription factor, down to the four factors responsible for reprogramming. However, retroviral insertions were variable in number for each factor when introduced in this manner. To better control copy number in the genome, all four factors could be introduced on one vector using a $2 \mathrm{~A}$ protease cleavage sequence to separate the different genes [31, 32]. Using a non-viral method simplified and reduced the cost of producing the four-factor vector. Additionally, transgene-free iPS cells would be more desirable than those still harboring the transgene insertions because reactivation of the inserted genes could result in oncogenic transformation [33]. piggyBac has been demonstrated to have a particular advantage in this area because the reprogramming factors, when introduced on a transposon, can be precisely excised from the iPSCs by re-expression of the piggyBac transposase. iPSC clones from which the reprogramming factors were excised were found to have an un-manipulated genome lacking any apparent mutations from the initial four-factor gene insertion [7, 8, 34]. Such "transgene-free" iPSCs will likely be needed for eventual therapeutic application, as accidental activation of the inserted transgenes could result in oncogenic transformation of differentiated iPSCs. piggyBac has successfully been used to generate iPSCs from a variety of species [7, 20, 34-36]. Recently, piggyBac was used to excise the selection component of a gene-targeting cassette in human iPSCs derived from patients with a1-antitrypsin deficiency. Zinc-finger nucleases were used to target integration of a therapeutic cassette into the a1-antitrypsin locus. A piggyBac transposon used for selection was subsequently excised, leaving behind gene repair with precise removal of the selection cassette [36]. 


\section{Modification of HSCs}

piggyBac has also been used for genetic modification of HSCs. Grabundjia et al. compared native piggyBac to $S B 100 X$, currently the most hyperactive version of the Sleeping Beauty transposase [37]. Although $S B 100 X$ appeared more active in this cell type, both systems were capable of modifying HSCs [37]. Recently, a hyperactive piggyBac transposase has been generated which appears more active than $S B 100 X$ in other cell types [38] but a comparison of hyperactive piggyBac to SB100X in HSCs has not yet been reported.

\section{Transposon-modified T cells}

Human T lymphocytes are an attractive cell type for adoptive immunotherapy for cancer. Retroviruses have been most widely used for genetic modification of $\mathrm{T}$ cells for adoptive transfer in clinical trials. Transposon-mediated gene modification of human $\mathrm{T}$ cells was first reported using Sleeping Beauty [39]. Recently, a human clinical trial has been approved for Sleeping Beauty transposon modification of T cells to target CD19-positive lymphoma [40]. The piggyBac transposon system has also been successfully utilized for gene-modification of human T cells. Stable transgene expression of up to $\sim 40 \%$ without selection was achieved using piggyBac followed by multiple logs of expansion of primary T-cells in culture [41]. piggyBac could simultaneously express multiple transgenes from two-independent transposons in combination with magnetic beads-selection for the transgenic surface marker, truncated CD19, in $\sim 85 \% \mathrm{~T}$ cells for over 9 weeks [41]. We used piggyBac to deliver a large $14 \mathrm{~kb}$ transposon in T-cells and to deliver a non-immunogenic suicide gene, inducible caspase 9 [41]. In human T-cells, integration site mapping showed that piggyBac did not preferentially integrate into or near known proto-oncogenes [42]. Hypothetically, piggyBac should be safer than the widely used retroviruses because piggyBac has less of a preference for proto-oncogenes and there have been no genotoxic events observed when using retroviruses in humans in $\mathrm{T}$ cell modification. Human $\mathrm{T}$-cells that were modified using piggyBac-transposons to express a chimeric antigen receptor targeting the CD19 antigen effectively killed CD19-expressing human lymphoma cell lines, demonstrating the functional activity of piggyBac-modified T-cells [43].

Virus-specific $\mathrm{T}$ cells have been shown to persist long-term in humans after genetic modification ex vivo followed by infusion [44]. piggyBac was used to genetically modify Epstein Barr Virus-specific cytotoxic T lymphocytes to target HER2 expressing cancer cells both in vitro and in vivo [45]. Additionally, piggyBac has recently been used to engineer Tcells to be resistant to rapamycin via delivery of a large mTOR transgene, a strategy which could be used in combination with rapamycin therapy for lymphoma [46]. Therefore, piggyBac has enabled high gene transfer efficiency in human T-cells and the ability to expand cells to clinical numbers. The unique features of piggyBac allow us to transfer large segments of DNA and to co-deliver and co-express multiple transgenes. The ability to deliver a suicide gene that can be selectively activated for ablation of all of the introduced $e x$ vivo-modified cells should improve safety. In pre-clinical models, piggyBac gene-modified human T-cells performed targeted killing of cancer cells in vitro and in vivo. piggyBac seems to be a promising non-viral gene delivery system for modifying $\mathrm{T}$ cells for cancer immunotherapy in a future clinical trial. 


\section{In vivo gene transfer}

Transposon systems have been used for in vivo gene transfer in mice, including correction of inherited diseases [47]. Hydrodynamic tail vein injection, which targets mouse liver, has been used to deliver piggyBac transposons containing reporter genes in vivo (Figure 2) [48, 49]. Long-term inducible gene expression in vivo has been observed after a single hydrodynamic injection of transposon DNA [49]. In addition to hydrodynamic injection, piggyBac transposons have been delivered to the mouse lung in polyethylenamine complexes and achieved long-term gene expression from the piggyBac transposons [49]. By modifying hydrodynamic injection to specifically target the kidneys of live mice, multiple transgenes have been delivered to the kidney [50,51]. Kidney-targeted delivery of piggyBac transposons carrying either glutathione S-transferase A4 [51] or insulin-like growth factor-1 receptor [50] gave significant protection against obstruction-induced renal fibrosis.

More recently, investigators have demonstrated phenotypic correction of hemophilia A [52] and B [53] by piggyBac-mediated gene transfer to mouse liver. One report coupled the use of piggyBac with novel synthetic cell-type-specific promoters to improve efficiency of gene expression in vivo [53]. Others have created hybrid adenovirus and adeno-associated virus (AAV) piggyBac vectors capable of in vivo gene transfer [54, 55]. Such vectors can overcome the limitations of delivering plasmid DNA into tissues by harnessing viruses to gain entry into the desired cell-type of interest.

\section{Special considerations of piggyBac}

\section{piggyBac vs nuclease platforms}

Given the recent advances of precision genome engineering including zinc finger nucleases, transcription activator-like effector nucleases (TALENs) and Cas9:sgRNA endonucleases [56], why would one consider using piggyBac for genome modification? All nuclease platforms appear to require homologous recombination for gene repair or transgene insertion. Homologous recombination usually has poor efficiency for gene transfer or modification in adult somatic quiescent tissues because the cellular homologous recombination machinery is not present. In adult tissues, non-homologous end-joining is preferred instead, resulting in disruptive mutations that result in loss of gene function, making gene addition strategies for somatic gene therapy perhaps unattainable with the nucleases.

piggyBac transposase is an enzyme which actively integrates DNA cargo without the need for cellular cofactors. However, integration of therapeutic genes inserted in the genome can potentially cause mutagenesis and impair the integrity of the genome. The level of gene expression in a gene-addition strategy may exceed endogenous levels, though cell-typespecific promoters can be used. Nuclease-based homologous recombination can edit the genome at a precise location to attain restored gene expression under endogenous regulation. However, nuclease platforms must be carefully titrated to avoid toxicity and off-target effects. For example, Cas9:sgRNA has been shown to result in chromosomal translocations in some instances [57]. piggyBac offers an adaptable system for co-delivery of one or multiple transgenes including other elements such as those required for inducible 
expression. There are also clear advantages of piggyBac for gene discovery or to generate stable cell lines.

\section{Precise transposon excision}

The piggyBac transposon system is unique in that it performs precise excision, leaving behind no footprint mutation. Precise excision was first demonstrated in insect cells [58] and subsequently in mammalian and human cells [5]. This characteristic of piggyBac has been used to generate transgene-free iPSCs, whereby transgenes are delivered for reprogramming on piggyBac transposons [7, 8, 34]. The piggyBac transposase can be re-expressed after reprogramming has occurred and the transgene cassettes can be excised from the genome, leaving behind no trace of the reprogramming elements or other genomic alterations. Other transposon systems such as Sleeping Beauty leave behind a footprint mutation [1, 59]. For gene-correction in human iPSCs, investigators have used a piggyBac selection transposon to enable selection of cells corrected by zinc finger nucleases. Subsequently, the piggyBac selection transposon was precisely excised, thereby removing the selection cassette at the site of gene repair [36]. Investigators have created a piggyBac transposase capable of excision but lacking integration (Exc+/Int-, Table 1) properties for seamless excision and removal of integrated piggyBac transposons [60].

\section{piggyBac fusion proteins}

The piggyBac transposase appears to be more amenable to protein-domain addition than the Sleeping Beauty transposase. This was initially tested by adding a hemagglutinin epitope tag for detection of expression which revealed no altered activity with piggyBac compared to Sleeping Beauty [5]. This flexibility of the transposase has been utilized to create an inducible transposase enzyme whereby transposition is regulated by tamoxifen [61]. piggyBac has also been fused to DNA binding domains to the transposase enzyme with the goal of redirecting piggyBac integration at the genomic level [62, 63], including to identify transcription factor binding sites in cultured cells [64]. Maintenance of transposase activity may be dependent on the terminus and linker(s) used to create the fusion protein, as well as the protein to be fused, so creation of novel fusions requires rigorous validation that they are functional [65].

The ability to direct transposon integration into user-defined chromosomal locations should provide more specificity in gene transfer experiments and improve safety in gene therapy experiments. Investigators first demonstrated the ability to target piggyBac integrations in a plasmid-based assay in mosquito embryos [66]. Subsequent investigations have demonstrated the ability of piggyBac to achieve site-directed integration in plasmid assays in human cells and in the human genome [62, 67]. piggyBac has shown biased integration into artificial target loci integrated in human cells [62]. Fusion of the piggyBac transposase to a Gal4 DNA binding domain biased piggyBac integration within $0.8 \mathrm{~kb}$ of the 56,898 putative Gal4 DNA binding sites in the human genome at a rate of $24 \%$ compared to $5 \%$ of native piggyBac integrations within the same window [67]. Recently, investigators have fused the transposase to a TAL domain and isolated single-copy integration clones with directed transposition at the CCR5 genomic locus observing an integration rate of between 0.01$0.015 \%$ depending on the TAL utilized [68]. The ability of a DNA binding domain to 
redirect piggyBac has also been used to "tag" transcription factor binding sites in mammalian cells [64]. Although the piggyBac transposase can be modified to achieve sitedirected integration, there is room for improvement. The transposase can still integrate transposon DNA at sites that are independent of the attached DNA-binding domain. Mutagenesis strategies aimed at making transposase integration activity more dependent on fused DNA binding domains may improve specificity.

\section{Control over transposon copy number}

The piggyBac transposon system appears titratable, whereby the user can change the amounts of transposon and transposase plasmids provided to achieve as few (as little as one) or as many integrations per cell as is desired [18]. Naturally, if plasmid amounts are lowered to achieve fewer integrations per cell, this will likely result in a lower percentage of total cells transfected. On the opposite end of the spectrum, the ability to achieve multiple integrations per cell has been exploited for multiplexed gene delivery. piggyBac was used to simultaneously deliver $\sim 28 \mathrm{~kb}$ of DNA on three different transposons creating stable cell lines expressing functional human brain sodium channels with the goal of using these cells for drug discovery [23]. The ability to integrate multiple transposon cargos simultaneously has enabled the creation of stable cell lines that could not be created previously and should open new avenues of research evaluating multi-protein complexes or signaling pathways.

\section{Improved piggyBac mutants}

Hyperactive piggyBac elements have also been generated. Investigators have improved activity through both transposase and transposon modifications. epiggyBac was generated by transposon inverted repeat mutation [69]. More recently, piggyBac was shown to be able to transpose in yeast and this was exploited to use random mutagenesis to create hyperactive transposases (HyPBase) in yeast [70]. HyPBase contains seven mutations and had increased transposition in mouse ES cells. A follow up study showed that HyPBase increased transposition activity in human cells, in vivo in mice, and in primary human $\mathrm{T}$ cells, and this head-to-head comparison with SB100X found HyPBase to be more active in human cells and in vivo in mice [38].

\section{Unanswered questions}

\section{Will piggyBac prove safe enough for clinical gene transfer applications?}

Insertional gene transfer carries with it the possible risk of insertional mutagenesis. Investigators have evaluated the potential for clonal outgrowth in long-term cultures of both piggyBac-modified human T lymphocytes [45] and human foreskin fibroblasts [71] and no clonal expansion has been observed. Additionally, there has been no observable tumor formation in the livers of wild-type mice modified with piggyBac out to one year in a small number of animals [49]. Nonetheless, with appropriate engineering of the transposon vector, insertional mutagenesis can be used to discover novel genes involved in a particular biological pathway. Long-term in vivo experimentation is necessary to determine the potential for genotoxicity when using piggyBac for potential gene therapy. 
piggyBac has different genomic target selectivity when integrating into genomic DNA when compared to other transposon systems such as Sleeping Beauty [17, 72-74]. It has been demonstrated that Sleeping Beauty exhibits a more random integration pattern whereas piggyBac exhibits more of a preference for gene containing regions and transcriptional start sites. Therefore, piggyBac may result in more reliable transgene expression; however, intragenic integrations could be detrimental to the host genome. Some investigators have altered piggyBac integration site selectivity at the genomic level by mutagenesis of the transposase [75]. This altered integration site selectivity at the genomic level was revealed by deep sequencing of thousands of recovered integration sites [75]. Additionally, further refinements could alter the genomic integration pattern of piggyBac to make it safer.

\section{Does plasmid backbone integration occur?}

Recently, a relatively high rate of plasmid backbone DNA integration when using piggyBac for genetic modification of human embryonic kidney (HEK-293) cells [71]. As piggyBac is highly active in HEK-293 cells, it remains to be determined if this phenomenon occurs in clinically relevant cell types such as human T lymphocytes or in vivo such as in mouse liver. The potential safety concern of the plasmid backbone randomly integrating into the host DNA remains to be determined, but can be nearly eliminated by cell sorting to remove cells expressing GFP from the integrated backbone [71]. Nonetheless, it remains to be determined if plasmid backbone integration occurs in clinically relevant cell types or in vivo animal models.

\section{Will targeting of piggyBac improve selectivity and efficiency enough for gene therapy?}

Investigators have demonstrated the ability to bias piggyBac integrations into user-defined genomic elements by fusing the transposase to ZFP or TAL proteins [62, 67, 68]. However, thus far, a high degree of specificity and selectivity in targeting integrations with piggyBac has not been demonstrated. Perhaps further refinement of the transposase or transposon could improve targeting specificity and efficiency. Targeting piggyBac integrations would greatly improve the safety of using this vector system for therapeutic gene transfer applications, especially for inherited diseases which will likely require a high degree of specificity in genome alteration with regards to safety.

\section{Concluding remarks and future perspectives}

The non-viral piggyBac transposon system has opened new areas of research with clinical implications. Undoubtedly, more studies will continue to be reported using piggyBac for gene discovery in cancer and other fields of research. The ability to create transgene-free iPSCs should improve the safety of their use in clinical practice. The use of piggyBac to efficiently modify human $\mathrm{T}$ cells may make immunotherapy applications more widely available due to its reduced cost as compared with viral vectors. Newer hyperactive piggyBac elements could increase efficiency even further. Finally, improving the targeting of piggyBac elements to user-defined chromosomal locations should improve safety and specificity in gene transfer approaches. piggyBac vector improvements and new delivery methodologies, whether viral or non-viral, should permit new biotechnology applications for transposon technology for pre-clinical and clinical applications. 


\section{Acknowledgments}

This work was supported by National Institutes Health grant R01-DK093660 (to MHW) and Department of Veterans Affairs grants I01-BX002190 (to MHW) and IK2-BX002797 (LEW).

\section{Glossary}

Transposon

Transgene

Seamless

excision

Targeted

integration transposons are self-mobilizing pieces of nucleic acids. In this article we are referring to a specific subclass of transposons, the DNA transposons that employ a "cut and paste" non-replicating mechanism of transposition. When the transposase is expressed, the transposon is excised from its current location (plasmid, virus, or genomic DNA harboring the transposon) and moves (or transposes) to a new location. Therefore, transposons can be used to transfer genes into a cell or tissue (by using plasmid or viral DNA to carry the transposon into the cell) or to move transposons throughout the genome (such as in gene discovery applications).

the transposon can be engineered to carry one or more gene(s) of interest which can be inserted into the genomic DNA. The transgene typically carries its own promoter. Alternatively, the transgene can be replaced with a gene trapping cassette for gene discovery. A wide variety of DNA sequences can be inserted into the transposon sequence for multiple different applications.

after piggyBac excises the transposon from DNA, it seamlessly generates the original piggyBac target site more than $95 \%$ of the time. This characteristic of piggyBac has allowed it to be used to integrate DNA sequences (such as those harboring antibiotic resistance or transcription factors) which can then be removed by re-expression of the transposase.

efforts are underway to target piggyBac integration into user-defined chromosomal loci. Native piggyBac integration is not targeted or sitespecific. Targeting piggyBac integration would improve its safety and efficiency in gene transfer applications.

\section{REFERENCES}

[1]. Ivics Z, Hackett PB, Plasterk RH, Izsvak Z. Molecular reconstruction of Sleeping beauty, a Tc1like transposon from fish, and its transposition in human cells. Cell. 1997; 91(4):501-10. [PubMed: 9390559]

[2]. Cary LC, Goebel M, Corsaro BG, Wang HG, Rosen E, Fraser MJ. Transposon mutagenesis of baculoviruses: analysis of Trichoplusia ni transposon IFP2 insertions within the FP-locus of nuclear polyhedrosis viruses. Virology. 1989; 172(1):156-69. [PubMed: 2549707]

[3]. Fraser MJ, Cary L, Boonvisudhi K, Wang HG. Assay for movement of Lepidopteran transposon IFP2 in insect cells using a baculovirus genome as a target DNA. Virology. 1995; 211(2):397407. [PubMed: 7645244]

[4]. Ding S, Wu X, Li G, Han M, Zhuang Y, Xu T. Efficient transposition of the piggyBac (PB) transposon in mammalian cells and mice. Cell. 2005; 122(3):473-83. [PubMed: 16096065] 
[5]. Wilson MH, Coates CJ, George AL Jr. PiggyBac Transposon-mediated Gene Transfer in Human Cells. MolTher. 2007; 15(1):139-45.

[6]. Mitra R, Fain-Thornton J, Craig NL. piggyBac can bypass DNA synthesis during cut and paste transposition. EMBO J. 2008; 27(7):1097-109. [PubMed: 18354502]

[7]. Woltjen K, Michael IP, Mohseni P, Desai R, Mileikovsky M, Hamalainen R, et al. piggyBac transposition reprograms fibroblasts to induced pluripotent stem cells. Nature. 2009; 458:766-70. [PubMed: 19252478]

[8]. Yusa K, Rad R, Takeda J, Bradley A. Generation of transgene-free induced pluripotent mouse stem cells by the piggyBac transposon. NatMethods. 2009; 6(5):363-9.

[9]. Rad R, Rad L, Wang W, Cadinanos J, Vassiliou G, Rice S, et al. PiggyBac transposon mutagenesis: a tool for cancer gene discovery in mice. Science. 2010; 330(6007):1104-7. [PubMed: 20947725]

[10]. Albieri I, Onorati M, Calabrese G, Moiana A, Biasci D, Badaloni A, et al. A DNA transposonbased approach to functional screening in neural stem cells. JBiotechnol. 2010; 150(1):11-21. [PubMed: 20688113]

[11]. You L, Chang D, Du HZ, Zhao YP. Genome-wide screen identifies PVT1 as a regulator of Gemcitabine sensitivity in human pancreatic cancer cells. BiochemBiophysResCommun. 2011; 407(1):1-6.

[12]. Wang W, Bradley A, Huang Y. A piggyBac transposon-based genome-wide library of insertionally mutated Blm-deficient murine ES cells. Genome Res. 2009; 19(4):667-73. [PubMed: 19233961]

[13]. Kong J, Wang F, Brenton JD, Adams DJ. Slingshot: a PiggyBac based transposon system for tamoxifen-inducible 'self-inactivating' insertional mutagenesis. Nucleic Acids Res. 2010; 38(18):e173. [PubMed: 20688953]

[14]. Wu S, Ying G, Wu Q, Capecchi MR. Toward simpler and faster genome-wide mutagenesis in mice. NatGenet. 2007; 39(7):922-30.

[15]. Landrette SF, Cornett JC, Ni TK, Bosenberg MW, Xu T. piggyBac transposon somatic mutagenesis with an activated reporter and tracker (PB-SMART) for genetic screens in mice. PLoS One. 2011; 6(10):e26650. [PubMed: 22039523]

[16]. Rad R, Rad L, Wang W, Strong A, Ponstingl H, Bronner IF, et al. A conditional piggyBac transposition system for genetic screening in mice identifies oncogenic networks in pancreatic cancer. Nat Genet. 2015; 47(1):47-56. [PubMed: 25485836]

[17]. Liang Q, Kong J, Stalker J, Bradley A. Chromosomal mobilization and reintegration of Sleeping Beauty and PiggyBac transposons. Genesis. 2009; 47(6):404-8. [PubMed: 19391106]

[18]. Wang W, Lin C, Lu D, Ning Z, Cox T, Melvin D, et al. Chromosomal transposition of PiggyBac in mouse embryonic stem cells. ProcNatlAcadSciUS A. 2008; 105(27):9290-5.

[19]. Jang CW, Behringer RR. Transposon-mediated transgenesis in rats. CSHProtoc. 2007; $2007 \mathrm{db}$.

[20]. Jiang MG, Li T, Feng C, Fu R, Yuan Y, Zhou Q, et al. Generation of transgenic rats through induced pluripotent stem cells. The Journal of biological chemistry. 2013; 288(38):27150-8. [PubMed: 23926100]

[21]. Wu Z, Xu Z, Zou X, Zeng F, Shi J, Liu D, et al. Pig transgenesis by piggyBac transposition in combination with somatic cell nuclear transfer. Transgenic research. 2013; 22(6):1107-18. [PubMed: 23857557]

[22]. Li Z, Zeng F, Meng F, Xu Z, Zhang X, Huang X, et al. Generation of transgenic pigs by cytoplasmic injection of piggyBac transposase-based pmGENIE-3 plasmids. Biology of reproduction. 2014; 90(5):93. [PubMed: 24671876]

[23]. Kahlig KM, Saridey SK, Kaja A, Daniels MA, George AL Jr. Wilson MH. Multiplexed transposon-mediated stable gene transfer in human cells. ProcNatlAcadSciUS A. 2010; 107(4): 1343-8.

[24]. Moriarity BS, Rahrmann EP, Keng VW, Manlove LS, Beckmann DA, Wolf NK, et al. Modular assembly of transposon integratable multigene vectors using RecWay assembly. Nucleic Acids Res. 2013; 41(8):e92. [PubMed: 23444141] 
[25]. Li Z, Michael IP, Zhou D, Nagy A, Rini JM. Simple piggyBac transposon-based mammalian cell expression system for inducible protein production. Proc Natl Acad Sci U S A. 2013; 110(13): 5004-9. [PubMed: 23476064]

[26]. Balasubramanian S, Matasci M, Kadlecova Z, Baldi L, Hacker DL, Wurm FM. Rapid recombinant protein production from piggyBac transposon-mediated stable $\mathrm{CHO}$ cell pools. $\mathrm{J}$ Biotechnol. 2015; 200:61-9. [PubMed: 25758242]

[27]. Tanaka A, Woltjen K, Miyake K, Hotta A, Ikeya M, Yamamoto T, et al. Efficient and reproducible myogenic differentiation from human iPS cells: prospects for modeling Miyoshi Myopathy in vitro. PLoS One. 2013; 8(4):e61540. [PubMed: 23626698]

[28]. Rostovskaya M, Fu J, Obst M, Baer I, Weidlich S, Wang H, et al. Transposon-mediated BAC transgenesis in human ES cells. Nucleic Acids Res. 2012; 40(19):e150. [PubMed: 22753106]

[29]. Takahashi K, Tanabe K, Ohnuki M, Narita M, Ichisaka T, Tomoda K, et al. Induction of pluripotent stem cells from adult human fibroblasts by defined factors. Cell. 2007; 131(5):86172. [PubMed: 18035408]

[30]. Yu JY, Vodyanik MA, Smuga-Otto K, ntosiewicz-Bourget J, Frane JL, Tian S, et al. Induced pluripotent stem cell lines derived from human somatic cells. Science. 2007; 318(5858):1917-20. [PubMed: 18029452]

[31]. Sommer CA, Stadtfeld M, Murphy GJ, Hochedlinger K, Kotton DN, Mostoslavsky G. Induced pluripotent stem cell generation using a single lentiviral stem cell cassette. Stem Cells. 2009; 27(3):543-9. [PubMed: 19096035]

[32]. Kim SI, Oceguera-Yanez F, Hirohata R, Linker S, Okita K, Yamada Y, et al. KLF4 N-Terminal Variance Modulates Induced Reprogramming to Pluripotency. Stem Cell Reports. 2015; 4(4): 727-43. [PubMed: 25772473]

[33]. Okita K, Ichisaka T, Yamanaka S. Generation of germline-competent induced pluripotent stem cells. Nature. 2007; 448(7151):313-7. [PubMed: 17554338]

[34]. Kaji K, Norrby K, Paca A, Mileikovsky M, Mohseni P, Woltjen K. Virus-free induction of pluripotency and subsequent excision of reprogramming factors. Nature. 2009; 458:771-5. [PubMed: 19252477]

[35]. Nagy K, Sung HK, Zhang P, Laflamme S, Vincent P, gha-Mohammadi S, et al. Induced pluripotent stem cell lines derived from equine fibroblasts. Stem Cell Rev. 2011; 7(3):693-702. [PubMed: 21347602]

[36]. Yusa K, Rashid ST, Strick-Marchand H, Varela I, Liu PQ, Paschon DE, et al. Targeted gene correction of alpha(1)-antitrypsin deficiency in induced pluripotent stem cells. Nature. 2011; 478(7369):391. + [PubMed: 21993621]

[37]. Grabundzija I, Irgang M, Mates L, Belay E, Matrai J, Gogol-Doring A, et al. Comparative analysis of transposable element vector systems in human cells. MolTher. 2010; 18(6):1200-9.

[38]. Doherty JE, Huye LE, Yusa K, Zhou L, Craig NL, Wilson MH. Hyperactive piggyBac Gene Transfer in Human Cells and In Vivo. HumGene Ther. 2012; 23(3):311-20.

[39]. Huang X, Wilber AC, Bao L, Tuong D, Tolar J, Orchard PJ, et al. Stable gene transfer and expression in human primary T cells by the Sleeping Beauty transposon system. Blood. 2006; 107(2):483-91. [PubMed: 16189271]

[40]. Hackett PB, Largaespada DA, Cooper LJ. A transposon and transposase system for human application. MolTher. 2010; 18(4):674-83.

[41]. Nakazawa Y, Huye LE, Dotti G, Foster AE, Vera JF, Manuri PR, et al. Optimization of the PiggyBac transposon system for the sustained genetic modification of human T lymphocytes. JImmunother. 2009; 32(8):826-36. [PubMed: 19752751]

[42]. Galvan DL, Nakazawa Y, Kaja A, Kettlun C, Cooper LJ, Rooney CM, et al. Genome-wide mapping of PiggyBac transposon integrations in primary human T cells. JImmunother. 2009; 32(8):837-44. [PubMed: 19752750]

[43]. Manuri PV, Wilson MH, Maiti SN, Mi T, Singh H, Olivares S, et al. piggyBac transposon/ transposase system to generate CD19-specific T cells for the treatment of B-lineage malignancies. HumGene Ther. 2010; 21(4):427-37. 
[44]. Heslop HE, Slobod KS, Pule MA, Hale GA, Rousseau A, Smith CA, et al. Long-term outcome of EBV-specific T-cell infusions to prevent or treat EBV-related lymphoproliferative disease in transplant recipients. Blood. 2010; 115(5):925-35. [PubMed: 19880495]

[45]. Nakazawa Y, Huye LE, Salsman VS, Leen AM, Ahmed N, Rollins L, et al. PiggyBac-mediated cancer immunotherapy using EBV-specific cytotoxic T-cells expressing HER2-specific chimeric antigen receptor. MolTher. 2011; 19(12):2133-43.

[46]. Huye LE, Nakazawa Y, Patel MP, Yvon E, Sun J, Savoldo B, et al. Combining mTor inhibitors with rapamycin-resistant T cells: a two-pronged approach to tumor elimination. MolTher. 2011; 19(12):2239-48.

[47]. Yant SR, Meuse L, Chiu W, Ivics Z, Izsvak Z, Kay MA. Somatic integration and long-term transgene expression in normal and haemophilic mice using a DNA transposon system. Nature Genetics. 2000; 25(1):35-41. [PubMed: 10802653]

[48]. Nakanishi H, Higuchi Y, Kawakami S, Yamashita F, Hashida M. piggyBac transposon-mediated long-term gene expression in mice. MolTher. 2010; 18(4):707-14.

[49]. Saridey SK, Liu L, Doherty JE, Kaja A, Galvan DL, Fletcher BS, et al. PiggyBac transposonbased inducible gene expression in vivo after somatic cell gene transfer. MolTher. 2009; 17(12): 2115-20.

[50]. Liang M, Woodard LE, Liang A, Luo J, Wilson MH, Mitch WE, et al. Protective Role of InsulinLike Growth Factor-1 Receptor in Endothelial Cells against Unilateral Ureteral ObstructionInduced Renal Fibrosis. Am J Pathol. 2015

[51]. Liang A, Wang Y, Woodard LE, Wilson MH, Sharma R, Awasthi YC, et al. Loss of glutathione S-transferase A4 accelerates obstruction-induced tubule damage and renal fibrosis. JPathol. 2012; 228:448-58. [PubMed: 22711583]

[52]. Matsui H, Fujimoto N, Sasakawa N, Ohinata Y, Shima M, Yamanaka S, et al. Delivery of fulllength factor VIII using a piggyBac transposon vector to correct a mouse model of hemophilia A. PLoS One. 2014; 9(8):e104957. [PubMed: 25126862]

[53]. Di Matteo M, Samara-Kuko E, Ward NJ, Waddingon SN, McVey JH, Chuah MK, et al. Hyperactive piggyBac transposons for sustained and robust liver-targeted gene therapy. Molecular therapy : the journal of the American Society of Gene Therapy. 2014; 22(9):1614-24. [PubMed: 25034357]

[54]. Cooney AL, Singh BK, Sinn PL. Hybrid Nonviral/Viral Vector Systems for Improved piggyBac DNA Transposon In Vivo Delivery. Mol Ther. 2015; 23:667-74. [PubMed: 25557623]

[55]. Smith RP, Riordan JD, Feddersen CR, Dupuy AJ. A hybrid adenoviral vector system achieves efficient long-term gene expression in the liver via piggyBac transposition. Hum Gene Ther. 2015

[56]. Kim H, Kim JS. A guide to genome engineering with programmable nucleases. Nature reviews Genetics. 2014; 15(5):321-34.

[57]. Frock RL, Hu J, Meyers RM, Ho YJ, Kii E, Alt FW. Genome-wide detection of DNA doublestranded breaks induced by engineered nucleases. Nat Biotechnol. 2015; 33(2):179-86. [PubMed: 25503383]

[58]. Fraser MJ, Ciszczon T, Elick T, Bauser C. Precise excision of TTAA-specific lepidopteran transposons piggyBac (IFP2) and tagalong (TFP3) from the baculovirus genome in cell lines from two species of Lepidoptera. Insect MolBiol. 1996; 5(2):141-51.

[59]. Liu GY, Aronovich EL, Cui ZB, Whitley CB, Hackett PB. Excision of Sleeping Beauty transposons: parameters and applications to gene therapy. Journal of Gene Medicine. 2004; 6(5): 574-83. [PubMed: 15133768]

[60]. Li X, Burnight ER, Cooney AL, Malani N, Brady T, Sander JD, et al. piggyBac transposase tools for genome engineering. Proc Natl Acad Sci U S A. 2013; 110(25):E2279-87. [PubMed: 23723351]

[61]. Cadinanos J, Bradley A. Generation of an inducible and optimized piggyBac transposon system. Nucleic Acids Res. 2007; 35(12):e87. [PubMed: 17576687]

[62]. Kettlun C, Galvan DL, George AL Jr. Kaja A, Wilson MH. Manipulating piggyBac transposon chromosomal integration site selection in human cells. MolTher. 2011; 19(9):1636-44. 
[63]. Wu SC, Meir YJ, Coates CJ, Handler AM, Pelczar P, Moisyadi S, et al. piggyBac is a flexible and highly active transposon as compared to sleeping beauty, Tol2, and Mos1 in mammalian cells. Proc Natl Acad Sci USA. 2006; 103(41):15008-13. [PubMed: 17005721]

[64]. Wang H, Mayhew D, Chen X, Johnston M, Mitra RD. "Calling Cards” for DNA-Binding Proteins in Mammalian Cells. Genetics. 2012; 190(3):941-9. [PubMed: 22214611]

[65]. Wilson MH, George AL Jr. Designing and testing chimeric zinc finger transposases. Methods MolBiol. 2010; 649:353-63.

[66]. Maragathavally KJ, Kaminski JM, Coates CJ. Chimeric Mos1 and piggyBac transposases result in site-directed integration. FASEB J. 2006; 20(11):1880-2. [PubMed: 16877528]

[67]. Owens JB, Urschitz J, Stoytchev I, Dang NC, Stoytcheva Z, Belcaid M, et al. Chimeric piggyBac transposases for genomic targeting in human cells. Nucleic Acids Res. 2012; 40(14):6978-91. [PubMed: 22492708]

[68]. Owens JB, Mauro D, Stoytchev I, Bhakta MS, Kim MS, Segal DJ, et al. Transcription activator like effector (TALE)-directed piggyBac transposition in human cells. Nucleic Acids Res. 2013; 41(19):9197-207. [PubMed: 23921635]

[69]. Lacoste A, Berenshteyn F, Brivanlou AH. An efficient and reversible transposable system for gene delivery and lineage-specific differentiation in human embryonic stem cells. Cell Stem Cell. 2009; 5(3):332-42. [PubMed: 19733544]

[70]. Yusa K, Zhou L, Li MA, Bradley A, Craig NL. A hyperactive piggyBac transposase for mammalian applications. ProcNatlAcadSciUS A. 2011; 108(4):1531-6.

[71]. Saha S, Woodard LE, Charron EM, Welch RC, Rooney CM, Wilson MH. Evaluating the potential for undesired genomic effects of the piggyBac transposon system in human cells. Nucleic Acids Res. 2015; 43(3):1770-82. [PubMed: 25605795]

[72]. Li X, Ewis H, Hice RH, Malani N, Parker N, Zhou L, et al. A resurrected mammalian hAT transposable element and a closely related insect element are highly active in human cell culture. Proc Natl Acad Sci USA. 2013; 110(6):E478-E87. [PubMed: 23091042]

[73]. Woodard LE, Li X, Malani N, Kaja A, Hice RH, Atkinson PW, et al. Comparative analysis of the recently discovered hAT transposon TcBuster in human cells. PLoSOne. 2012; 7(11):e42666.

[74]. Li MA, Pettitt SJ, Eckert S, Ning Z, Rice S, Cadinanos J, et al. The piggyBac transposon displays local and distant reintegration preferences and can cause mutations at noncanonical integration sites. MolCell Biol. 2013; 33(7):1317-30.

[75]. Burnight ER, Staber JM, Korsakov P, Li X, Brett BT, Scheetz TE, et al. A Hyperactive Transposase Promotes Persistent Gene Transfer of a piggyBac DNA Transposon. Molecular therapy Nucleic acids. 2012; 1:e50. [PubMed: 23344650]

[76]. Li MA, Turner DJ, Ning Z, Yusa K, Liang Q, Eckert S, et al. Mobilization of giant piggyBac transposons in the mouse genome. Nucleic Acids Res. 2011; 39(22):e148. [PubMed: 21948799]

[77]. Izsvak Z, Ivics Z, Plasterk RH. Sleeping Beauty, a wide host-range transposon vector for genetic transformation in vertebrates. Journal of Molecular Biology. 2000; 302(1):93-102. [PubMed: 10964563]

[78]. Mates L, Chuah MK, Belay E, Jerchow B, Manoj N, costa-Sanchez A, et al. Molecular evolution of a novel hyperactive Sleeping Beauty transposase enables robust stable gene transfer in vertebrates. NatGenet. 2009; 41:753-61. 




For illustration purposes, transposition is demonstrated from plasmid DNA into the genome, although it could also be from one genomic locus to another. Once transposase is expressed (red circles) it binds to the piggyBac inverted repeats (IRs) and induces nicking and 3' hydrophilic attack of the TTAA ends. Hairpin formation occurs with transposon excision. The transposon is then integrated and joined into genomic DNA at a TTAA nucleotide sequence resulting in TTAA target site duplication at the genomic locus. If precise excision is desired, transposase can be re-expressed and the transposon can be excised, thereby recreating the original TTAA target site at the original locus. 


\section{Box 2: Seamless excision of piggyBac transposon, a unique tool for genetic manipulation}

The piggyBac transposon integrates into a TTAA nucleotide sequence $>95 \%$ of the time. Upon integration, target site-duplication occurs with a TTAA flanking both ends of the transposon DNA. Upon excision, the original TTAA target site is reformed $>95 \%$ of the time. This seamless excision activity has led to in some clever genome engineering approaches. piggyBac can integrate transcription factor cassettes for cellular reprogramming into iPS cells. These factors may later be removed after re-expression of the transposase resulting in iPS cells with "unaltered" genomic DNA [8]. Others have performed gene editing of a disease locus in human cells and were able to select genomemodified cells with a piggyBac transposon selection cassette [30]. Once cells were selected and verified, the piggyBac transposon was excised, resulting in corrected human cells without additional genome modifications such as a remnant selection cassette. 


\section{Box 3: Generation of stable cell lines for drug discovery}

Integrating viral vectors, such as retroviruses and lentiviruses, have limited cargo capacity (Table). In contrast, plasmid-based piggyBac has a large cargo capacity up to several hundred kilobases [76]. Multiplexed gene integration with piggyBac permits the design of novel cell-based assays for drug discovery, whether the target is a multi-protein complex such as a G-protein coupled receptor or a multi-subunit ion channel complex [23]. The ability to use a plasmid-based system to create stable cell lines expressing multiple transgenes should improve upon current drug discovery methodology. One can more easily generate stable cell lines expressing one or more transgenes which can be evaluated in high throughput screening assays to derive novel targets for potential therapies. piggyBac has also facilitated inducible large-scale protein production from human cells [25] for production of therapeutic enzymes, hormones, or antibodies. 


\section{Highlights}

- The piggyBac transposon system offers a versatile genome modification system for biotechnology applications.

- piggyBac can be used for gene discovery or transgene insertion in vitro or in vivo.

- Stable cell lines can be more easily generated with piggyBac as compared to other gene delivery systems.

- New therapeutic strategies should result from piggyBac-related gene transfer research. 


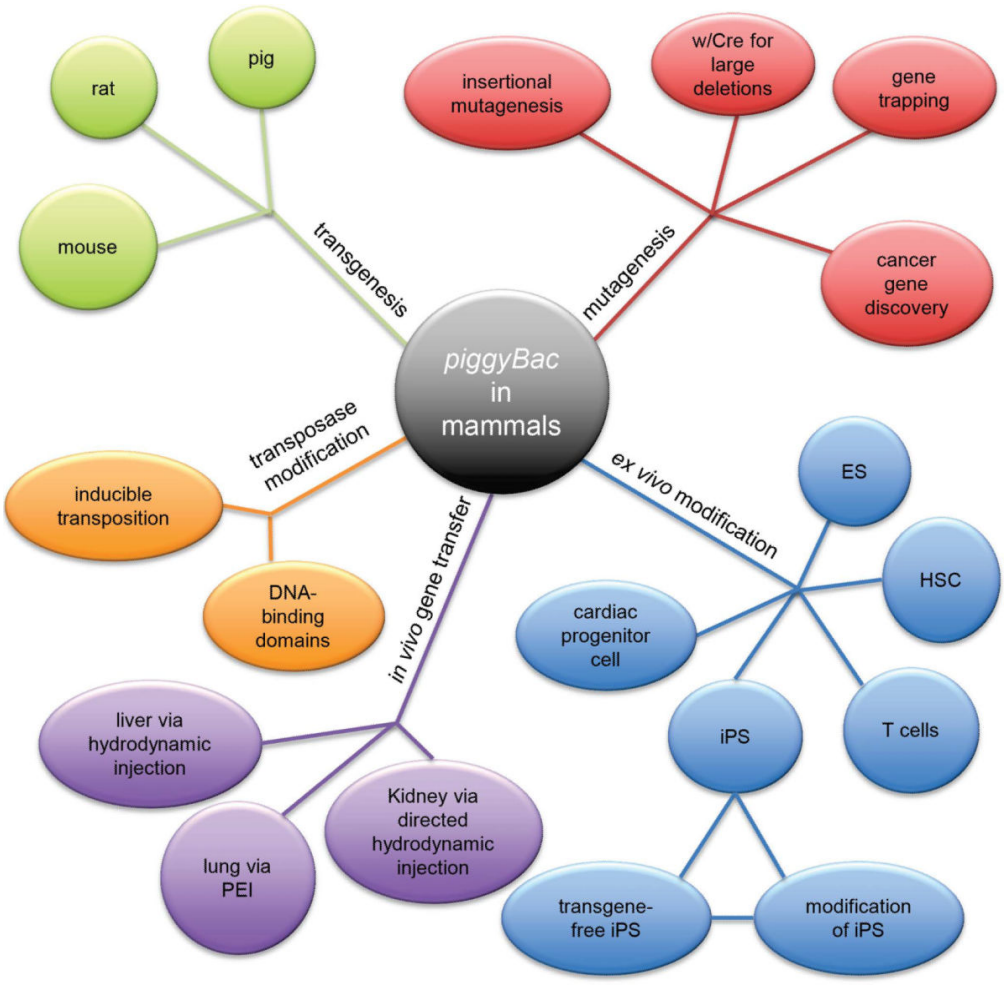

Figure 1.

The versatility of piggyBac in biotechnology applications for modification of the mammalian genome. piggyBac has been effective for mutagenesis in both cell lines and mice, transgenesis in a variety of species, modification of clinically relevant cell types, and in vivo gene transfer. The transposase is also amenable to modification to create an inducible transposon system or fusion to DNA binding domains or other protein sequences. 
A



B
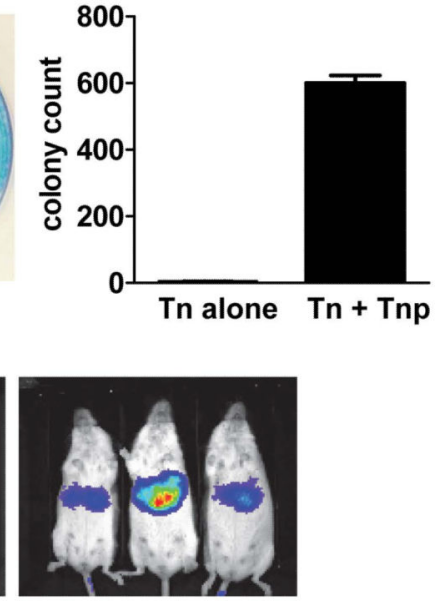

Figure 2.

piggyBac activity in cultured cells and in vivo. (A) Left, HEK-293 cells were transfected with a plasmid containing an antibiotic selection transposon without (left) or with (right) transposase. Two days after transfection, cells were split into the antibotic for selection and cultured for 2 weeks and finally fixed and stained with methylene blue for visualization. The left plate shows the traditional way of making stable cell lines (very inefficient). The right plate demonstrates the efficiency of piggyBac in making stable cell lines (highly efficient). Right, a colony count on antibiotic resistant cells derived from a dilution of the plates on the left (figures adapted from [23]). (B) Hydrodynamic tail vein injection was used to deliver luciferase transposons to the livers of mice without (left) or with (right) tranposase. in vivo imaging was then used to image luciferase expression at day 300 post gene transfer. Therefore, a single injection of piggyBac transposon DNA led to luciferase expression almost 1 year post gene transfer in a live animal (figure adapted from [49]). 


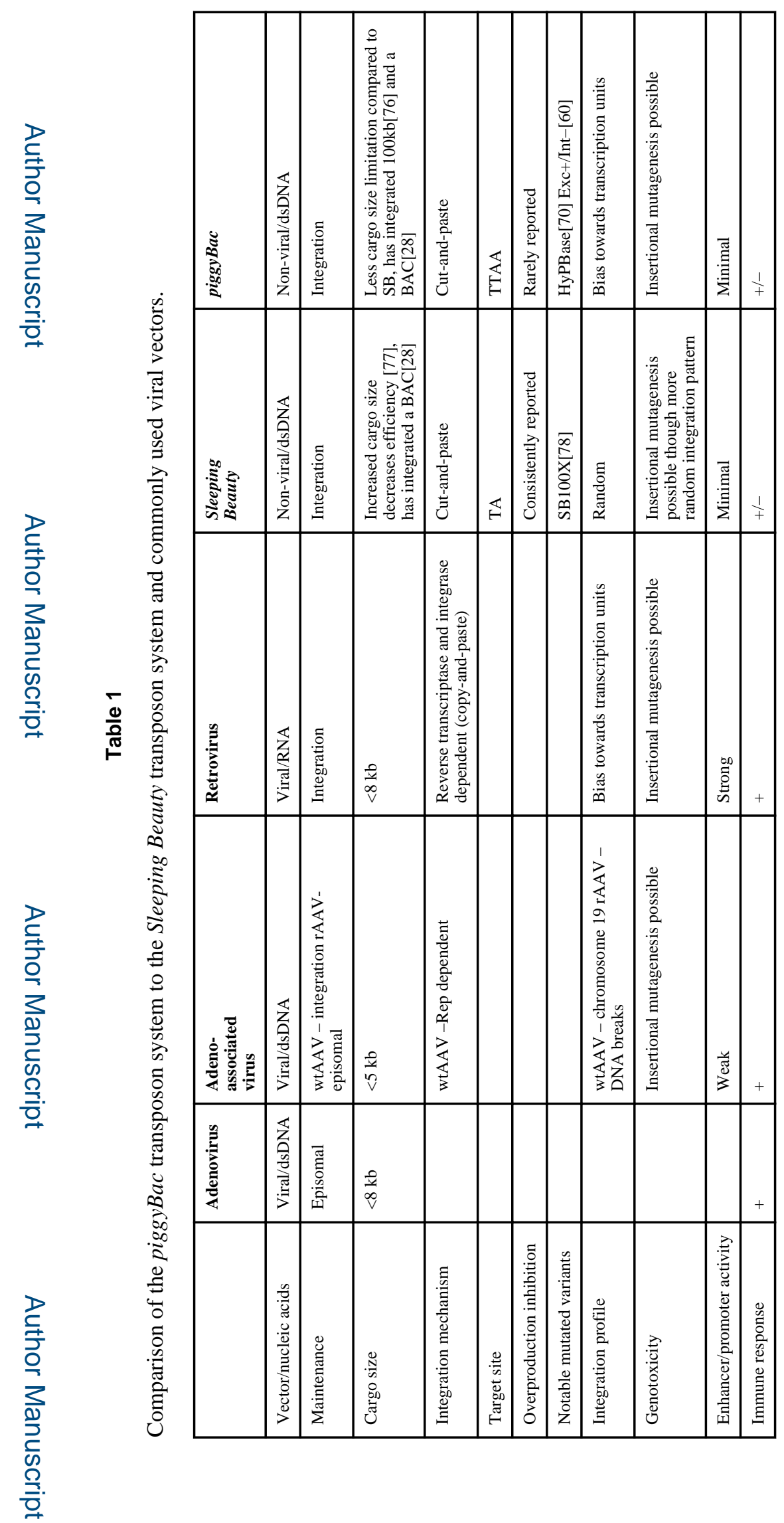

Trends Biotechnol. Author manuscript; available in PMC 2016 September 01. 